\title{
Instabilities of interacting vortex rings generated by an oscillating disk
}

\author{
Jian Deng* and Lubao Teng \\ State Key Laboratory of Fluid Power \& Mechatronic Systems, \\ Zhejiang University, Hangzhou 310027, People's Republic of China \\ Key Laboratory of Soft Machines and Smart Devices of Zhejiang Province, \\ Zhejiang University, Hangzhou 310027, People's Republic of China and \\ Department of Mechanics, Zhejiang University, Hangzhou 310027, People's Republic of China \\ C. P. Caulfield \\ BP Institute, University of Cambridge, Madingley Road, Cambridge CB3 OEZ, UK and \\ Department of Applied Mathematics and Theoretical Physics, University of Cambridge, \\ Centre for Mathematical Sciences, Wilberforce Road, Cambridge CB3 0WA, UK \\ Xuerui Mao \\ School of Engineering and Computer Science, Durham University, Durham DH1 3LE, UK
}

(Dated: July 5, 2016)

\begin{abstract}
We propose a novel yet natural model to probe in a controlled fashion the instability of interacting vortex rings shed from the edge of an oblate spheroid disk of major diameter $c$, undergoing oscillations of frequency $f_{0}$ and amplitude $A$. We perform a Floquet stability analysis to determine the characteristics of the instability modes, which depend strongly on the azimuthal (integer) wavenumber $m$. We vary two key control parameters, the Keulegan-Carpenter number $K C=2 \pi A / c$ and the Stokes number $\beta=f_{0} c^{2} / \nu$, where $\nu$ is the kinematic viscosity of the fluid. We observe two distinct flow regimes. First, for sufficiently small $\beta$, and hence low frequency of oscillation corresponding to relatively weak interaction between sequentially shedding vortex rings, symmetry breaking occurs directly to a single unstable mode with $m=1$. Second, for sufficiently large yet fixed values of $\beta$, corresponding to a higher oscillation frequency and hence stronger ring-ring interaction, the onset of asymmetry is predicted to occur due to two branches of high $m$ instabilities as the amplitude is increased, with $m=1$ structures only being dominant for sufficiently large values of $K C$. These two branches can be distinguished by the phase properties of the vortical structures above and below the disk. The region in $(K C, \beta)$ parameter space where these two high $m$ instability branches arise can be described accurately in terms of naturally defined Reynolds numbers, using appropriately chosen characteristic length scales. We subsequently carry out direct numerical simulations of the fully three-dimensional flow to verify the principal characteristics of the Floquet analysis, in particular demonstrating that high wavenumber symmetry-breaking generically occurs when vortex rings sequentially interact sufficiently strongly.
\end{abstract}

\section{INTRODUCTION}

Vortex rings are canonical fluid structures which arise in a wide range of different circumstances, over a huge variety of characteristic length scales. They have been observed in the smoke generated in volcanic eruptions [1, 2], in human hearts during cardiac relaxation [3], and in the propulsion of jellyfish [4], to name just a few examples. The characteristics of vortex rings have been considered at least since the 19th century, when Thomson [5] stated that the vortex ring in an ideal fluid was indestructible. However, it is now widely appreciated that vortex rings are prone to a rich variety of instabilities once a relatively low threshold in Reynolds number is crossed, and indeed, for sufficiently high Reynolds numbers, the vortex cores can be fully turbulent [6].

Consideration of instability and turbulent transition of vortex rings is an active area of research, not least because vortex rings, due to the inherent symmetry of their

\footnotetext{
* Corresponding author: zjudengjian@zju.edu.cn
}

characteristic state, allows the controlled investigation of the relative importance of vorticity distribution, intensity and flow curvature in the onset of instability. The instability of a vortex ring was first documented as early as 1939 [7], and there are now many known mechanisms by which a ring may become unstable. Indeed, in essence, the study of vortex ring instabilities falls into two generic categories. The first category consists of isolated instabilities of a vortex ring propagating through an otherwise quiescent fluid, where the so-called Moore-Saffman-TsaiWidnall (MSTW) instability is responsible for the development of unstable waves in the vortex core $[6,8-11]$. The MSTW instability was initially proposed by considering a straight vortex tube subjected to a straining field in a plane perpendicular to the tube axis [9], leading to the deformation of the initially circular vortex core into an ellipse. More recently, a new primary linear instability mode was found to be driven by the effect of the inherent curvature of a vortex ring $[12,13]$. A more detailed description of these two sources of isolated instabilities can be found in ref.[14], in which both the elliptical instability and the curvature instability were predicted by 
an asymptotic theory with two respective corrections. In laboratory experiments, vortex rings are commonly produced by pushing fluid out a tube or through a hole, commonly referred to as a 'vortex-ring gun'. In such experiments the dimensionless ratio of stroke length $L$ to exit diameter $D, L / D$, is a key parameter. For too large $L / D$, the vortex ring will be followed by a trailing jet, while for too small $L / D$, the vortex ring may not propagate away from the tube. In these experiments, azimuthal waves with wavenumber $m=8,9,12$ [7], $m=6,7$ [15], $m=9$ [16] have been recorded.

The second, and richer, category of vortex ring instability consists of coupled instabilities associated with the primary vortex ring undergoing an interaction, for example with another ring [17] or with a boundary such as a wall [18-20]. Such coupled instabilities are typically more complicated than the more studied isolated instabilities. For example, when a vortex ring impinges on a solid wall, a strong inviscid interaction occurs in the form of the ejection of secondary or indeed tertiary vortex rings from the boundary layer, with multiple azimuthal waves being observed both experimentally [18] and numerically $[19,21,22]$. In the case of vortex ringwall interaction, interactions with permeable walls have also been considered [23]. It has been observed that the presence of a permeable wall leads to significant changes in the flow field, and the final outcome of the interaction is itself strongly influenced by surface permeability. Indeed, under certain favourable conditions, the primary vortex ring was observed to pass through the permeable wall and continue as a modified vortex ring in its lee [20]. A vortex ring can also interact with another one, as can be observed in the head-on collision of two vortex rings. In this process, the two rings become unstable and reconnect to form a series of smaller rings, with the number ranging from 15 to 20 as the Reynolds number is increased from 860 to 1500 [17].

Unravelling the inherent flow instability characteristics of interacting vortex rings from the bulk flow evolution of the interaction itself is an important and challenging problem, particularly due to the inevitable timedependence of such interactions. Here we report a novel model which naturally enables the periodic generation of vortex rings in a well-controlled and repeatable fashion. These vortex rings inevitably interact, and so it is possible to consider subsequently the instability associated with ring-ring interactions in a controlled fashion. The flows considered here are also complementary to ringwall interactions, as the periodic generation of the vortex rings from an oscillating disk inevitably leads to a strong fluid-structure interaction. Our primary aim is to identify the circumstances in which high wavenumber asymmetric instabilities onset in ring-ring interactions in a context different from yet complementary to head-on collision. To achieve this aim, the rest of the paper is organised as follows. In section II we formulate our model, and present our numerical methodology. In section III, we then present our results, showing a pleas-

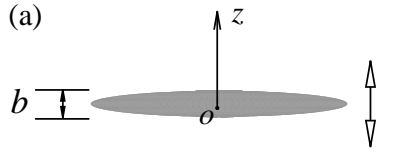

(b)

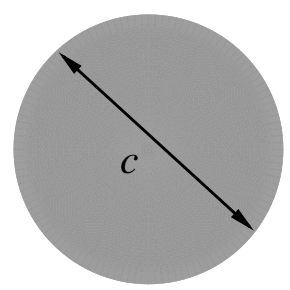

FIG. 1. Geometric definition of the disk (actually an oblate spheroid in rigorous geometric terms): (a) side view, where $z$ is the symmetry axis along which the disk oscillates, $b$ is the minor diameter, and the hollow arrow denotes the direction of oscillation; (b) top view, where $c$ is the major diameter of the spheroid.

ing correspondence between our Floquet stability analysis and nonlinear direct numerical simulations. Finally, in section IV, we draw our conclusions, and point towards further research directions.

\section{PROBLEM FORMULATION AND NUMERICAL METHODOLOGY}

\section{A. Definition of the problem studied}

The basic flow geometry is shown in Fig.1. Our model consists of a periodically oscillating oblate spheroid 'disk'. For simplicity, we fix the aspect ratio of the disk $A R=b / c=0.1$, where $b$ is the minor diameter and $c$ is the major diameter of the oblate spheroid. We oscillate the disk vertically with a frequency $f_{0}$ (or a period of $\left.T_{0}=1 / f_{0}\right)$ and amplitude $A$. This oscillation periodically sheds vortex rings from either side of the disk. The rings then propagate away from the disk, and interact with each other in a regular and controlled fashion. We investigate the linear stability properties and nonlinear evolution of such flows in an appropriately defined frequency-amplitude parameter space. The conventional non-dimensional frequency or the Stokes number $\beta$, and the non-dimensional amplitude or Keulegan-Carpenter number $K C$, are defined as

$$
\beta=\frac{f_{0} c^{2}}{\nu}, \quad K C=\frac{2 \pi A}{c},
$$

where $\nu$ is the kinematic viscosity of the fluid. We note that if we regard $c$ as the length scale and $f_{0} c$ as the characteristic velocity, $\beta$ is in the form of a Reynolds number, which is commonly used to characterize a fluid flow. However, as we will discuss below, the flow induced by an oscillating disk does not depend on this parameter alone, as the oscillation amplitude $A$, or its non-dimensional form $K C$, also plays an important role. 


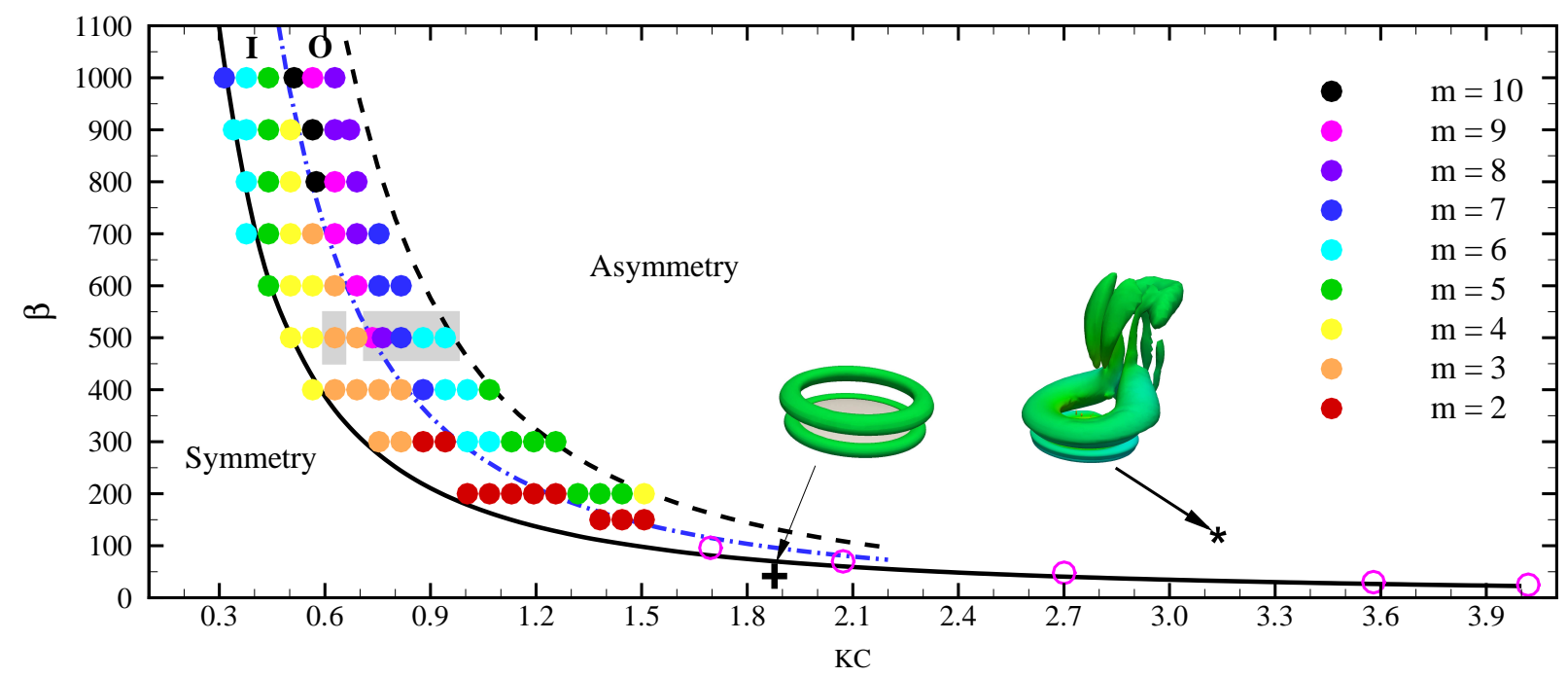

FIG. 2. (Color online) Floquet instabilities to the two-dimensional vortex ring flow. Neutral stability points are marked with open circles, and instabilities are represented by filled circles with various colors to distinguish their wavenumbers. The solid line, $R e_{L}=\beta K C^{3 / 2}=180$ as defined in (8) bounds the symmetry instability region $(m=0)$ to the lower-left. The dashed line, $R e_{H}=\beta \cdot K C^{2}=467$ as defined in (9) bounds the region to the upper-right of $m=1$ asymmetry from higher wavenumber $m>1$ modes in the high- $\beta$ regime. The instabilities between these two lines can be divided (by the empirical dot-dashed line, $\beta \cdot K C^{7 / 4}=312$ ) into two groups associated with the in-phase branch 'I' and the out-of-phase branch ' $\mathrm{O}$ ' of instability as shown in Fig.3, which plots the stability properties at $\beta=500$ (marked with shading) for various $K C$. Also shown are two three-dimensional structures of the vortex rings generated by an oscillating disk in the low- $\beta$ regime, visualized using $Q=20$ [24], obtained by solving the full nonlinear three-dimensional Navier-Stokes equations, colored by the pressure distribution: for a typical axisymmetric flow at $K C=1.88, \beta=50$ (marked by + ); and for a typical asymmetric flow with azimuthal wavenumber $m=1$ at $K C=3.14, \beta=100$ (marked by $\star$ ).

\section{B. Numerical methodology}

The flow induced by the vertical oscillation of a disk, sketched in Fig.1, can be simulated in a frame of reference fixed to the disk. Assuming that the fluid is Newtonian and incompressible, the flow is governed by the incompressible Navier-Stokes (NS) equations. An extra term is simply added to the right-hand side of the quations to account for the non-inertia of the reference frame. This approach is more efficient than employing a deforming mesh to tackle the disk oscillation, as has been used in previous studies [25-27]. This renders the system to be solved as

$$
\begin{gathered}
\boldsymbol{\nabla} \cdot \mathbf{u}=0 \\
\frac{\partial \mathbf{u}}{\partial t}=-\mathbf{u} \cdot \boldsymbol{\nabla} \mathbf{u}-\nabla p+\nu \nabla^{2} \mathbf{u}-\boldsymbol{a}
\end{gathered}
$$

where $\mathbf{u}$ and $p$ denote the velocity vector field and the modified scalar pressure (the real pressure divided by the uniform fluid density) field respectively and $t$ is the time. The boundary conditions in the non-inertial frame are such that the velocity is zero at the disk wall and, at the external boundaries of the domain, the vertical component is equal to the negative of the disk velocity.

Although we can also solve numerically the full unsteady, three-dimensional problem given by (2) and (3), we mainly focus on the global linear stability problem, which gives a efficient estimation of the azimuthal symmetry breaking of the vortex rings. The flow field is thus decomposed into the summation of an axisymmetric base flow and a perturbation, $(\mathbf{u}, p)=(\mathbf{U}, P)+\left(\mathbf{u}^{\prime}, p^{\prime}\right)$. The small-amplitude perturbation satisfies the unsteady Navier-Stokes equations linearized around the base state, namely

$$
\begin{gathered}
\boldsymbol{\nabla} \cdot \mathbf{u}^{\prime}=0, \\
\frac{\partial \mathbf{u}^{\prime}}{\partial t}=-\mathbf{U} \cdot \nabla \mathbf{u}^{\prime}-\mathbf{u}^{\prime} \cdot \nabla \mathbf{U}-\nabla p^{\prime}+\nu \nabla^{2} \mathbf{u}^{\prime} .
\end{gathered}
$$

Since the axisymmetric base flow has a natural periodicity, we conduct a Floquet stability analysis. We use a spectral-element method to calculate both the axisymmetric base flow and the instabilities [28]. Asymmetric Floquet modes are determined from the linearized Navier-Stokes equations assuming sinusoidal variation in the azimuthal direction with integer (due to the geometric periodicity) wavenumber $m$. Any perturbation at time $t=T$ can be decomposed as the sum of modes

$$
\mathbf{u}^{\prime}(r, \theta, z, t)=\sum_{m=0}^{+\infty} \mathbf{u}^{(m)}(r, z, t) e^{i m \theta},
$$

where $i=\sqrt{-1}$. To determine the structure of the most unstable mode and its growth rate at a given wavenumber 
$m$, we define a ( $T$-periodic) operator $\mathbf{A}$, which evolves a perturbation from $t=0$ to $t=T=2 \pi / f_{0}$ by integrating the Navier-Stokes equations, linearised about the calculated axisymmetric base flow, forward in time, and so:

$$
\mathbf{u}^{\prime}(T)=\mathbf{A}(T) \mathbf{u}^{\prime}(0)
$$

The Floquet modes and their multipliers, i.e. the growth rates over a period, can be obtained by calculating the eigenvectors and eigenvalues of $\mathbf{A}$, through applying an Arnoldi method to a Krylov subspace constructed by iteratively integrating the linearized Navier-Stokes equations. These eigenvalues are complex values and the dominant one, namely the one with the largest magnitude, denoted as $\mu_{\max }$, corresponds to the least stable mode. If $\left|\mu_{\max }\right|>1$, this mode is unstable and will exhibit perturbation energy growth.

Compromising between computational efficiency and accuracy in this study, we find that a grid consisting of approximately 800 quadrilateral elements resolves the flow field with an accuracy of $0.5 \%$. We minimise boundary effects by considering a computational domain with the far field boundary $25 c$ from the disk. Each spectral element is further decomposed into $64(8 \times 8)$ nodes through a spectral expansion. This code has been successfully applied to various flow problems [29-31].

To investigate the nonlinear development of the identified unstable Floquet modes, we use a finite volume numerical code to solve the fully nonlinear threedimensional Navier-Stokes equations. This code has been applied to both a flapping airfoil [32-34], and an oscillating elliptical foil [35]. We ensure that the Courant number of all cells is less than one, and that each oscillation period $T$ is decomposed into at least 2000 time steps so that the unsteadiness caused by the oscillation is well-resolved. Through extensive validation, we find that a mesh with approximately 4000000 cells resolves the three-dimensional flow structures adequately.

\section{RESULTS}

In this section we present the stability results obtained in the parametric space of $(K C, \beta)$ as shown in Fig.2, in which $\beta$ is varied in increments of $\triangle \beta=100$ (or 20 when $\beta \leq 150$ ), and $K C$ is varied in increments of $\triangle K C=$ 0.063 (or smaller increments within some distinct regimes to track the transitions more accurately). Moreover, to provide an intuitive understanding of the flow structure in its finite amplitude saturated state, three-dimensional direct numerical simulations (DNS) have also been carried out for $\beta=500,0.628 \leq K C \leq 0.942$.

\section{A. Stability characteristics}

The two-dimensional simulation of (2) and (3) is conducted for 50 cycles after which we find that the solution has become periodic. We then save a complete state vector of the flow over an oscillation period at time intervals separated by $T_{0} / 64$. In stability analyses, these saved flow vectors are used to generate the base flow at each time step using a third-order Lagrangian interpolation. We find that in all cases the critical instabilities are predicted to be non-oscillatory and non-degenerate, in that the first Floquet multiplier to cross the unit circle is always real and associated with a single wavenumber $m$. However, there is a qualitative variation in the behaviour with the flow parameters, particularly between flows with 'low' and 'high' frequency of oscillation, as shown in Fig.2. At lower Stokes numbers $\beta$, the axisymmetry breaks at a single wavenumber $m=1$. In the bottom right-hand corner of Fig.2, we show with a solid line the neutral stability curve in $(K C, \beta)$ space determined by Floquet analysis for 'low' $\beta \leq 130$, which separates symmetric flow to the left from asymmetric flow (with $m=1)$ to the right.

Two typical saturated three-dimensional simulations on either side of the neutral curve are also shown in Fig.2 when the disk has reached the lowest point in its trajectory. In the symmetric regime, there is a single vortex ring above the disk, while below the disk, the vortex ring shed at the previous half-period of the oscillation has already been completely dissipated due to the effect of viscosity, unsurprisingly because of the low frequency of oscillation. Indeed, the empirically-determined boundary between symmetric and asymmetric flow regime on the $(K C, \beta)$ space shown in Fig. 2 with a solid line may be interpreted as a critical low- $\beta$ Reynolds number, as the boundary corresponds to

$$
R e_{L}=\beta \cdot K C^{3 / 2}=\frac{(2 \pi)^{3 / 2} f_{0} A \sqrt{c A}}{\nu}=180 .
$$

For this flow regime, the appropriate length scale in the critical Reynolds number is the geometric mean of the oscillation amplitude and the disk diameter, as both quantities are significant to ensure the required flow interactions to trigger the observed instabilities.

At higher frequencies, or equivalently higher values of the Stokes number $\beta$, the stability properties are qualitatively different, as a regime of parameter space appears in which the onset of asymmetry is due to (in general) two distinct branches of higher wavenumber $m>1$ Floquet modes. Although the flow remains symmetric to the left of the solid line $R e_{L}=180$, at higher values of $\beta$ axisymmetry is broken by in general two different branches of higher wavenumber (i.e. $m>1$ ) modes as $K C$ increases, with dominant $m=1$ asymmetry only occurring to the right of the dashed line shown on Fig.2. This dashed line is defined by a different critical Reynolds number $R e_{H}$, defined as

$$
R e_{H}=\beta \cdot K C^{2}=\frac{(2 \pi)^{2} f_{0} A^{2}}{\nu}=467 .
$$

At such high values of $\beta$, the high frequency of oscillation suggests that the amplitude of the oscillation is the only 

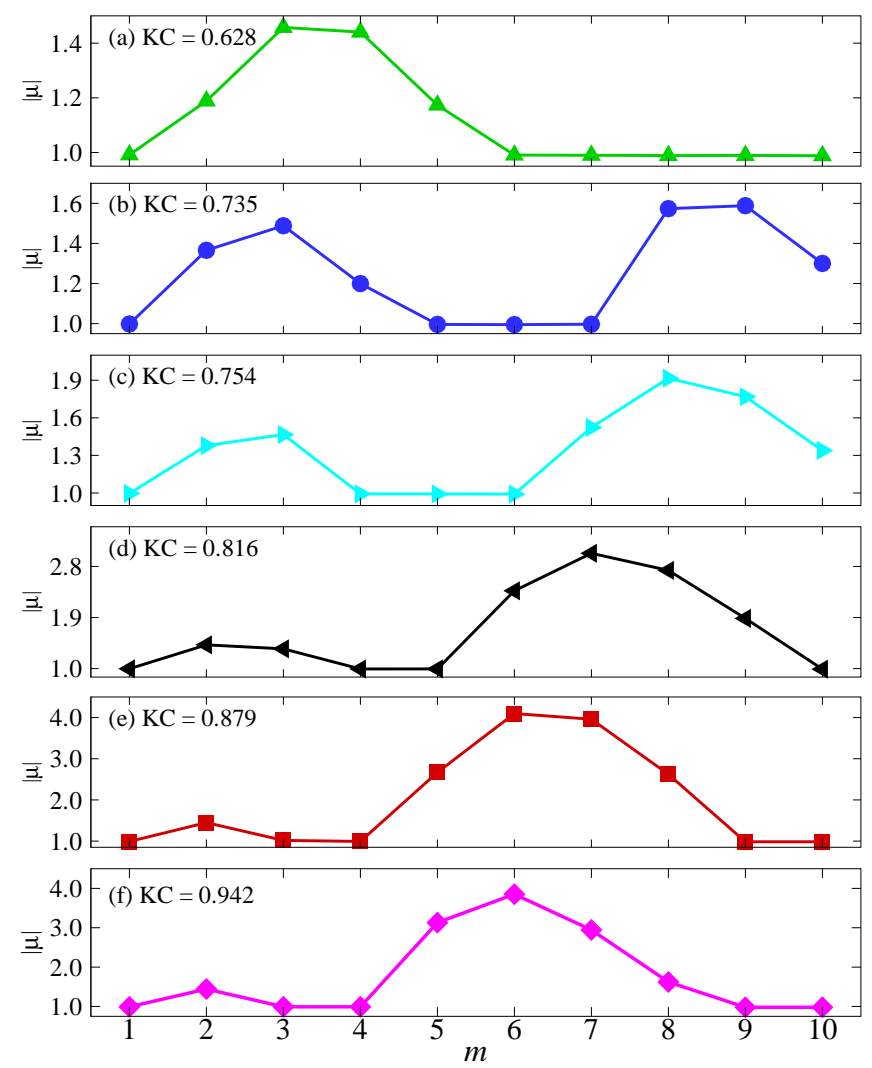

FIG. 3. (Color online) Variation of Floquet multiplier magnitude $\left|\mu_{m}\right|$ with azimuthal wavenumber $m$ for different $K C$ at $\beta=500$, as marked with shading on Fig.2. Note the two distinct branches of high wavenumber $m>1$ instability (subdivided by a dot-dashed line in Fig.2): the in-phase branch 'I' at lower $m$ and the out-of-phase branch 'O' at higher $m$.

key length scale, consistently with the structure of this high $\beta$ Reynolds number.

As shown in Fig.2, the stability properties between these two curves are non-trivial. At fixed $\beta$, as $K C$ increases through the critical value associated with $R e_{L}=$ 208, an unstable mode with $m>1$ appears, (shown with open symbols on Fig.2) with the value of $m$ increasing with $\beta$. At a fixed value of $\beta$, as $K C$ is increased, the value of $m$ associated with the most unstable mode typically decreases initially. However, there is typically a discontinuous increase in the azimuthal wavenumber (followed once again by a decrease with increasing $K C$ ) at some intermediate value, as marked by the dot-dashed curve on Fig.2.

Taking $\beta=500$ as an example, (as shown with shading on Fig.2) this behaviour at fixed $\beta$ as $K C$ is varied can be understood in terms of the relative importance of two distinct branches of instability identified by our Floquet analysis. In Fig.3, we plot the variation of the Floquet multiplier magnitude $\left|\mu_{m}\right|$ with wavenumber $m$ at $\beta=500$ for various $K C$. For this value of $\beta$, the critical value of $K C$ corresponding to $R e_{L}=180$

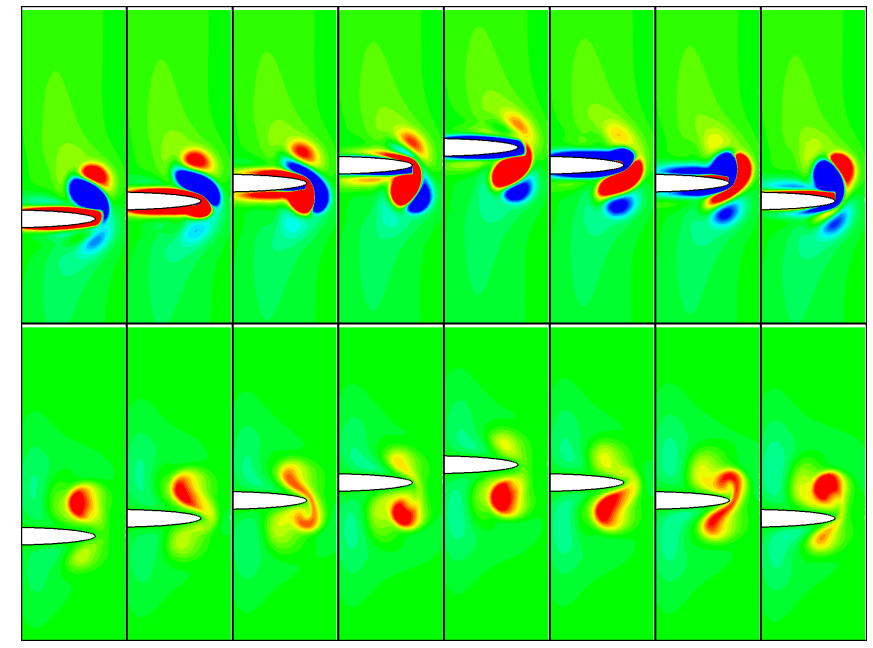

FIG. 4. (Color online) Evolution of the vortical field for the axisymmetric base flow (top row) and the $\theta$-component of the perturbation velocity for $m=4$ (bottom row) during a cycle for $\beta=500$ and $K C=0.628$. From left to right, the disk starts from its lowest position then moves upwards, and the images are evenly spaced by $T_{0} / 8$, where $T_{0}$ is the period of oscillation. Blue (dark gray) and red (light gray) colors denote negative values and positive values respectively.

is $K C_{L}=0.506$. For relatively small $K C>K C_{L}$, e.g. $K C=0.628$ as shown in Fig.3a, non-trivial growth rate occurs in the range of $m=1-5$, with maximum growth rate for this branch of instability being associated with an $m$ which decreases from 4 to 3 . However, as we increase $K C$, another branch of instability appears at higher $m=5 \sim 10$. The maximum growth rate for this branch is associated with an $m$ which decreases from 8 to 6 , although the lower $m$ and now sub-dominant branch of instability remains. This behaviour is perhaps not surprising, since it is not unusual for two distinct branches of instability associated with distinct wavenumber bands to coexist in certain parameter ranges. For example, two such branches are observed in the secondary transition in the wake behind a bluff body [36], with two modes of instabilities observed corresponding respectively to a short wavelength and a high wavelength.

To illustrate the characteristics of these two branches, we present the instantaneous vortical fields for the axisymmetric base flow and the $\theta$-component of the perturbation velocity at different phases in the base flow's period for the branch ' $\mathrm{I}$ ' in Fig.4, and for the branch ' $\mathrm{O}$ ' in Fig.5. First, we note that the flow is indeed periodic due to the periodic oscillation of the disk, which is apparently indicated by the evolution of the base flow within a cycle. As shown in the top row of Fig.4, as the disk moves upwards, the vortex (in blue color or dark gray) formed in the previous downstroke stage is shed to the lower side of the disk, and pairs with the newly formed vortex (in red color or light gray) into a vortex dipole. This process repeats during the downstroke. There are 


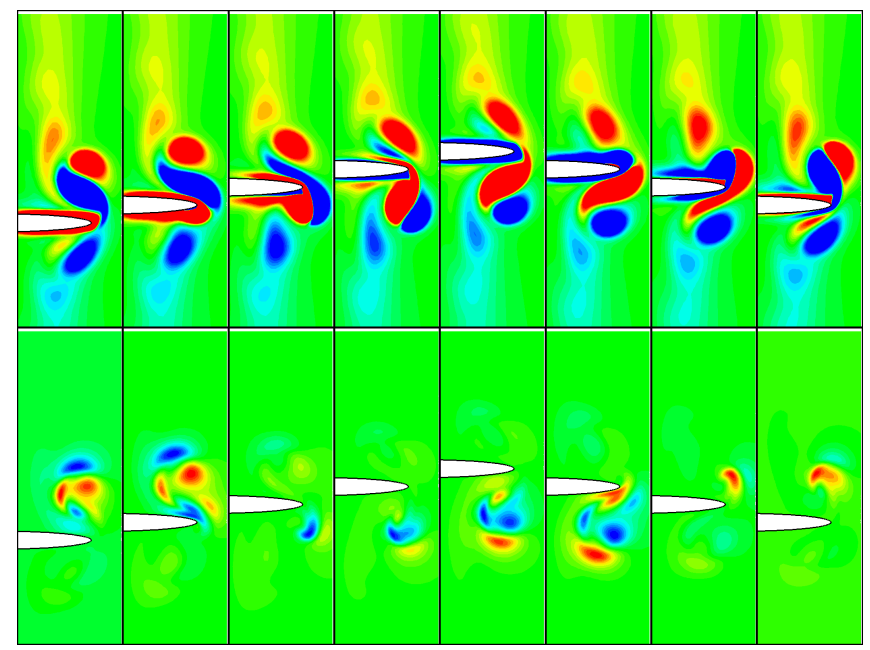

FIG. 5. (Color online) Evolution of the vortical field for the axisymmetric base flow (top row) and the $\theta$-component of the perturbation velocity for $m=7$ (bottom row) during a cycle for $\beta=500$ and $K C=0.816$. From left to right, the disk starts from its lowest position then moves upwards, and the images correspond to eight evenly spaced phases in a period of the disk oscillation. Blue (dark gray) and red (light gray) colors denote negative values and positive values respectively.

marked differences between Fig.4 and Fig.5 (for top rows) in both intensity and complexity of the vortices. In Fig.4, the vortices shed from the disk are immediately diffused as the disk reverses, while in Fig.5 they propagate further away from the disk. In the bottom rows of Fig.4 and Fig.5, the $\theta$-components of the perturbation velocity are normalized by the maximum value in the leftmost panel respectively for each figure. At the bottom of Fig.4, we observe that the perturbation is localized within the base flow vortices within the cycle. It is interesting to note that the $\theta$-component of the perturbation velocity keeps positive values during the whole cycle, implying that the resulting azimuthal waves below the disk propagate in phase with those above the disk. On the other hand, at the bottom of Fig.5, the perturbation field has very different characteristics. The perturbation field contains finer structures, and the $\theta$-component of the perturbation velocity during the upstroke and during the downstroke have opposite sign.

\section{B. Three-dimensional DNS results}

Floquet analysis has elucidated the stability characteristics of interacting vortex rings, and in particular predicted two distinctly different branches at sufficiently high $\beta$. To investigate these two high $m$ branches of instability further, and in particular to identify distinguishing characteristics between the two branches, we perform three-dimensional direct numerical simulations. Several oscillation periods are integrated to ensure that the flow has reached a nonlinear saturated state. We note that the three-dimensional direct numerical simulation results for small $\beta$ both before and after the symmetry breaking have been shown in Fig.2. In this section, we concentrate on the regime with high $\beta$. In Fig.6, we show the vortex structures at $\beta=500$ for different values of $K C$. Comparing the wave numbers of these structures with those of the Floquet modes (see Fig.3) we find a reasonable agreement for most cases, although for the cases at $K C=0.735$ and $K C=0.754$, (plotted in Fig.6(b) and (c)) the nonlinear flow appears to have locked on to the lower wavenumber branch instability, with $m=3$, as opposed to the higher wavenumber branch with $m=9$ and $m=8$ respectively, as shown in Fig.3(b) and (c). Possible reasonable explanations for this nonlinear discrepancy are that the higher wavenumber modes are more strongly affected by viscosity, or alternatively that the high-wavenumber modes saturate earlier and are overtaken by the more robust low-wavenumber modes during the flow's nonlinear development.

Physically the two different branches of instability have qualitatively different structures, as is apparent by comparison of Figs 6(a)-(c) and (d)-(f). For the modes associated with the lower wavenumber branch shown in Fig.6(a)-(c), the vortical structures which develop above and below the disk are synchronized 'in phase', in that the structures appear at the same azimuthal location. This finding accords well with the stability results shown in Fig.4. Therefore, we refer to this branch as branch 'I'. Conversely, for the modes associated with the higher wavenumber branch shown in Fig.6(d)-(f), the vortical structures which develop above and below the disk are aligned 'out of phase', in that the structures below the disk appear at azimuthal locations corresponding to the gaps between the structures above the disk, according well with the stability results shown in Fig.5. Therefore, we refer to this branch as branch ' $\mathrm{O}$ ', and it is clear that the two branches are distinct in both wavenumber range and physical nonlinear structure.

\section{CONCLUSIONS}

We have studied numerically the instabilities arising in the interacting vortex rings generated by an oscillating disk. We report that different regimes, characterizing the instabilities, can be identified in the $(K C, \beta)$ phase space. As we have pointed out, the $\beta$ parameter is in the form of a Reynolds number, although, it cannot determine the dynamics of this system completely, as the nondimensional amplitude, quantified by the Keulegan number $K C=2 \pi A / c$ is also important. Instead of using a single Reynolds number, in this paper we have found that two alternate definitions of Reynolds number are significant. First, for sufficiently small $\beta$, symmetry breaking occurs at a critical Reynolds number of $R e_{L}=180$, directly to a single unstable mode with $m=1$. Second, for sufficiently large yet fixed values of $\beta$, the onset of asymmetry is pre- 
(a)

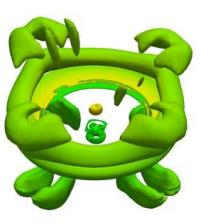

(b)

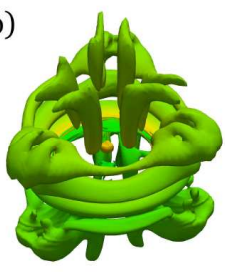

(c)

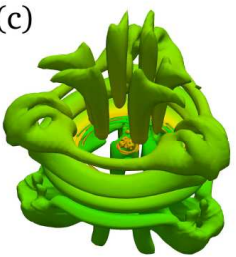

(d)

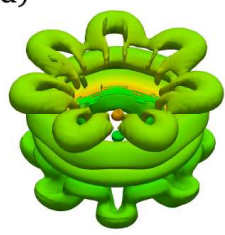

(e)

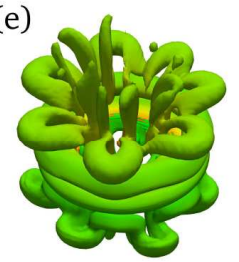

(f)

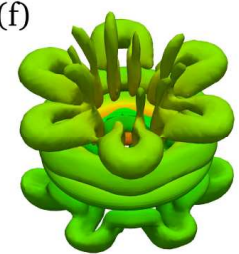

FIG. 6. (Color online) Three-dimensional structures of the vortex rings generated by an oscillating disk, visualized using $Q=20$ [24], obtained by solving the fully three-dimensional Navier-Stokes equations, colored by pressure, with $\beta$ fixed at $\beta=500$ while $K C$ varies. (a) $K C=0.628$, (b) $K C=0.735$, (c) $K C=0.754$, (d) $K C=0.816$, (e) $K C=0.879$, (f) $K C=0.942$. Note that the vortical structures above and below the disk are in phase for in (a)-(c) and out of phase in (d)-(f) corresponding to branch ' $\mathrm{I}$ ' and branch 'O' instabilities respectively.

dicted to occur due to two branches of high $m$ instabilities as the amplitude is increased, with $m=1$ structures only being dominant for sufficiently large values of $K C$, bounded by a critical Reynolds number of $R e_{H}=467$ in the upper-right-hand part of parameter space. Again, for high $\beta, R e_{L}=180$ bounds the symmetry region $(m=0)$ in the lower-left-hand part of parameter space.

We have focused on the regime of high $\beta$ between the two critical Reynolds numbers, in which organized unstable modes have been predicted by linear theory and realized by our three-dimensional direct numerical simulations. We have divided this regime into two branches. For the first branch 'I', the unstable waves arising in the vortex rings below the disk and above the disk are synchronized 'in phase', while for the secpmd branch ' $\mathrm{O}$ ' they are aligned 'out of phase'. We note that the unstable modes in branch 'I' with small wave numbers, e.g. $m=2$ or $m=3$ do not appear to have been physically observed, or numerically predicted previously.

Interestingly, our study suggests that very high wavenumber azimuthal instabilities arise in a relatively narrow part of parameter space, with the right balance between amplitude and frequency of the primary oscillation, and that a specific phase relationship between perturbations is most conducive to such high wavenumber instabilities. It remains an open question whether such instabilities can survive to even higher values of Stokes number $\beta$, and indeed it is unclear just how robust the predictions of linear stability theory are for strongly nonlinear flows. Nevertheless, our results suggest a clear and controlled method by which the break down of vortex rings to high wavenumber instabilities due to mutual interaction may be considered in detail, either experimentally or numerically, and so it seems natural to use this flow model to investigate this important problem further.

\section{ACKNOWLEDGMENTS}

This research is supported by the National Natural Science Foundation of China (Grant No: 11272283) and the Public Projects of Zhejiang Province (Grant No: 2015C31126) to conduct this research.
[1] F. I. Perret, Sci. Am. 75, 4 (1913).

[2] O. V. Fuentes, Eur. J. Mech. B. Fluids 43, 166 (2014).

[3] M.-A. Bray and J. P. Wikswo, Phys. Rev. Lett. 90, 238303 (2003).

[4] B. J. Gemmell, D. R. Troolin, J. H. Costello, S. P. Colin, and R. A. Satterlie, Journal of The Royal Society Interface 12, 20150389 (2015).

[5] W. Thomson, Lond. Edinb. Dubl. Phil. Mag. 34, 15 (1867).

[6] S. E. Widnall and C.-Y. Tsai, Philos. Trans. R. Soc. AMath. Phys. Eng. Sci. 287, 273 (1977).

[7] C.-H. Krutzsch, Annalen der Physik 427, 497 (1939).

[8] S. E. Widnall, D. B. Bliss, and C.-Y. Tsai, J. Fluid Mech. 66, 35 (1974).

[9] D. Moore and P. Saffman, in Proc. R. Soc. A-Math. Phys. Eng. Sci. (The Royal Society, 1975), vol. 346, pp. 413425.

[10] C.-Y. Tsai and S. E. Widnall, J. Fluid Mech. 73, 721 (1976).
[11] K. Shariff and A. Leonard, Annu. Rev. Fluid Mech. 24, 235 (1992).

[12] Y. Hattori and Y. Fukumoto, Phys. Fluids 15, 3151 (2003).

[13] Y. Fukumoto and Y. Hattori, J. Fluid Mech. 526, 77 (2005).

[14] F. J. Blanco-Rodríguez, S. Le Dizès, C. Selçuk, I. Delbende, and M. Rossi, J. Fluid Mech. 785, 219 (2015).

[15] S. E. Widnall and J. Sullivan, in Proceedings of the Royal Society of London A: Mathematical, Physical and Engineering Sciences (The Royal Society, 1973), vol. 332, pp. 335-353.

[16] I. S. Sullivan, J. J. Niemela, R. E. Hershberger, D. Bolster, and R. J. Donnelly, Journal of Fluid Mechanics 609, 319 (2008).

[17] T. Lim and T. Nickels, Nature 357, 225 (1992).

[18] J. Walker, C. Smith, A. Cerra, and T. Doligalski, J. Fluid Mech. 181, 99 (1987).

[19] M. Cheng, J. Lou, and L.-S. Luo, J. Fluid Mech. 660, 
430 (2010).

[20] M. Cheng, J. Lou, and T. Lim, Physics of Fluids (1994present) 26, 103602 (2014).

[21] P. Orlandi and R. Verzicco, J. Fluid Mech. 256, 615 (1993).

[22] H. Ren and X.-Y. Lu, Commun. Comput. Phys. 18, 1122 (2015).

[23] T. Lim and D. Adhikari, in Vortex Rings and Jets (Springer, 2015), pp. 33-60.

[24] J. Jeong and F. Hussain, J. Fluid Mech. 285, 69 (1995).

[25] H. M. Blackburn and R. D. Henderson, Journal of Fluid Mechanics 385, 255 (1999).

[26] J. S. Leontini, M. Thompson, and K. Hourigan, Journal of Fluid Mechanics 577, 79 (2007).

[27] R. d. S. Gioria, P. J. S. Jabardo, B. S. Carmo, and J. R. Meneghini, Journal of Fluids and Structures 25, 676 (2009).
[28] G. Karniadakis and S. Sherwin, Spectral/hp element methods for computational fluid dynamics (Oxford University Press, 2013).

[29] R. Natarajan and A. Acrivos, J. Fluid Mech. 254, 323 (1993).

[30] G. Sheard, M. Thompson, and K. Hourigan, J. Fluid Mech. 492, 147 (2003).

[31] G. Rocco, T. Zaki, X. Mao, H. Blackburn, and S. Sherwin, Aerosp. Sci. Technol. (2015).

[32] J. Deng, C. Caulfield, and X. Shao, Phys. Fluids 26, 043102 (2014).

[33] J. Deng and C. Caulfield, Phys. Rev. E 91, 043017 (2015)

[34] J. Deng, L. Sun, and X. Shao, Phys. Rev. E 92, 063013 (2015).

[35] J. Deng and C. Caulfield, J. Fluid Mech. 787, 16 (2016).

[36] G. E. Karniadakis and G. S. Triantafyllou, J. Fluid Mech. 238, 1 (1992). 